\title{
A Study on Vehicle Connectivity for VANETs Adopting Beamforming Antenna Array
}

\author{
Liu Yang, ${ }^{1,2}$ Weidong Xiang, ${ }^{3}$ and Guoxin Zheng ${ }^{1}$ \\ ${ }^{1}$ Key Laboratory of Specialty Fiber Optics and Optical Access Networks, Shanghai University, Shanghai 200072, China \\ ${ }^{2}$ School of Electronic and Information, Shanghai Dianji University, Shanghai 201306, China \\ ${ }^{3}$ College of Engineering and Computer Science, University of Michigan-Dearborn, Dearborn, MI 48128, USA
}

Correspondence should be addressed to Liu Yang; yangliu0806@126.com

Received 27 September 2016; Revised 7 March 2017; Accepted 27 March 2017; Published 6 April 2017

Academic Editor: M. Chadli

Copyright (c) 2017 Liu Yang et al. This is an open access article distributed under the Creative Commons Attribution License, which permits unrestricted use, distribution, and reproduction in any medium, provided the original work is properly cited.

\begin{abstract}
An analytical mobility model based on Erlang distribution is introduced to portray sparse and dense traffic scenarios in the distributions of time headway. Upon the mobility model, the connectivity probability is then derived in two-way highway when adopting store-forward strategy. Moreover, path losses with and without beamforming antenna array are compared to further study the connectivity under different traffic density scenarios. Meanwhile, the effectiveness of beamforming technology in Vehicular Ad hoc Networks (VANETs) is investigated and concluded through analytical study and simulations.
\end{abstract}

\section{Introduction}

Vehicular Ad hoc Networks (VANETs) are deployed along highways and local roads for exchanging traffic information among vehicles to achieve the enhanced safety and comfort [1]. Vehicles are connected via multiple technologies of vehicle-to-vehicle (V2V) communications based on Wi-Fi, ZigBee, and Bluetooth over $2.4 \mathrm{GHz}$ and Dedicated Short Range Communication (DSRC) over 5.9 GHz [2]. Network connectivity is an important topic for VANETs, and several metrics are adopted to portray the connectivity of vehicles in literatures $[3,4]$. Two models are typically adopted in the study on connectivity: (1) mobility model and (2) channel model.

In general, it is not trivial to build an analytical mobility model to accurately describe the real-world vehicle behaviors under sparse and dense traffic [5]. To respond to this end, a queuing model based on Erlang distribution is adopted to depict the vehicle behavior, which is mathematically represented by the distribution of time headway.

In previous literatures, a unit disk channel model was commonly adopted where two vehicles are regarded as connected if their distance is less than a given threshold, otherwise not. Such a channel model is a way to be realistic since channel characteristics are decisive to the performance of vehicular system [6]. In this paper, path loss is calculated according to an enhanced channel model presented in this paper.

$\mathrm{V} 2 \mathrm{~V}$ communications exchange information among vehicles and help to reduce accidents and assuage congestion. Enhancing the vehicle connectivity for VANETs is quite meaningful. Currently, omnidirectional or wide beam antenna is widely used in $\mathrm{Wi}-\mathrm{Fi}, \mathrm{ZigBee}$, Bluetooth, and DSRC terminals, whereas poor gain performance especially in long-distance scenarios becomes the vital fault. To solve this problem, beamforming antenna array is suggested in this paper to enhance connectivity of VANETs through adaptively adjusting radiated direction to achieve high gains and large coverages.

\section{Related Work}

A good number of mobility models for VANETs have been researched through analytical studies or experiments in the past. Some work assumes that distances between adjacent vehicles are exponentially distributed in both streets and other situations [7-9]. In [10], mobility model is approximated by an exponential distribution with parameters extracted from data captured on I-80 highway in California under sparse traffic. Those assumptions are reasonable when 
traffic is light, but they do not fit well with diverse traffic conditions. In [11], a Gaussian-exponential mixture model is proposed to characterize the distance between vehicles in a highway. In [12], mobility scenarios are defined by using a set of traces from traffic count on two highways around Madrid in Spain. This research indicates that mobility models changed by time during a day. Up until now, the suitability and accuracy of mobility model in the scale of days have not been presented in the past under sparse and dense traffic. Therefore, a new model is proposed to represent the distributions of time headway under diverse traffic in a scale ranging from hours to days. We adopt Erlang distribution which is presented by the distribution of the time headway, to depict the behavior of vehicles in sparse and dense scenarios $[13,14]$, evoked by the fact that Erlang model is used to characterize call holding time in communications networks $[15,16]$.

Moreover, some literatures simply adopt a unit disk channel model leading to oversimplified connection assumption between vehicles $[8,12]$. In this paper, the free-space path loss model based on FRIIS equation is introduced to solve such an issue [17]. Meanwhile, some studies tried to improve connectivity as follows. In [18], the connectivity probability between vehicles and the infrastructure is improved through multihop broadcasting in infrastructure-based Vehicular Networks. Grouping vehicles into platoons is another approach to improve connectivity and reduce fuel consumption in [19]. In this paper, the improvement of the connectivity of VANETs is considered by employing beamforming antenna array on vehicles. Beamforming is a technique of signal directional transmission to achieve enhanced gain and enlarged coverage. It also benefits from power assumption and security. In [20], the impact of different antenna patterns on vehicular network is simulated in the contexts of collision avoidance.

To summarize, in this paper the mobility model is presented to portray diverse traffic density in a scale ranging from hours to days, and beamforming antenna array is considered to improve the connectivity of VANETs.

\section{Systems Models}

The mobility model and channel model used in this paper are described as follows.

3.1. Mobility Model. A mobility model based on Erlang distribution is introduced to present the distribution of the time headway, $t$, as below. At first, the probability density function (PDF) of Erlang distribution is written as

$$
f(t ; a, q)=\frac{(q a)^{a}}{(a-1) !} \times t^{a-1} e^{-a q t}, \quad t>0,
$$

where $q$ is the rate of flow, a positive value, and also known as the reciprocal of the mean time headway. $a$ is the shape parameter, which is an integer. Different traffic density can be illustrated by corresponding values of $a$. Its cumulative

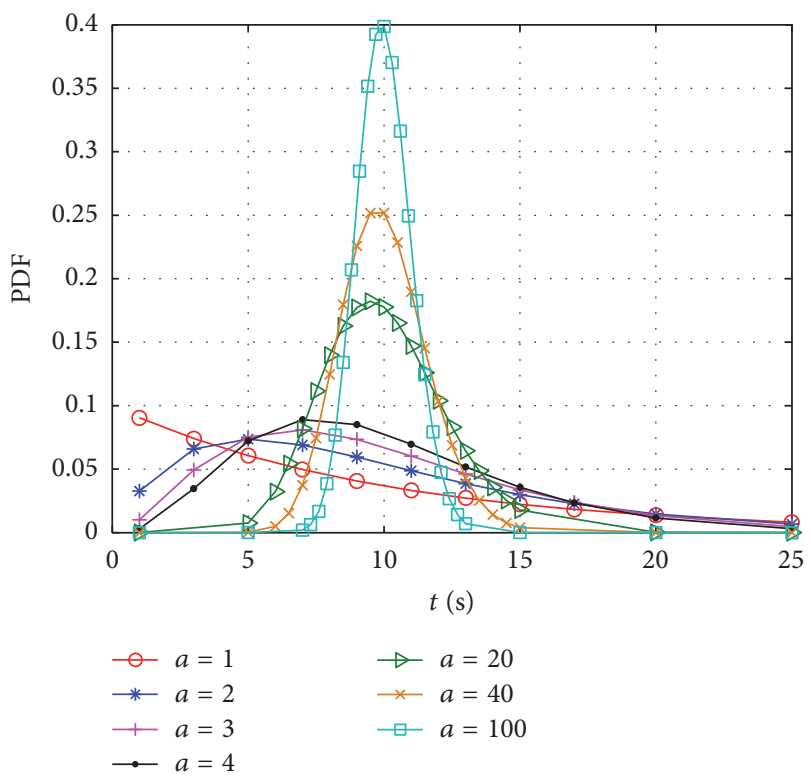

FIgURE 1: The PDFs of mobility model.

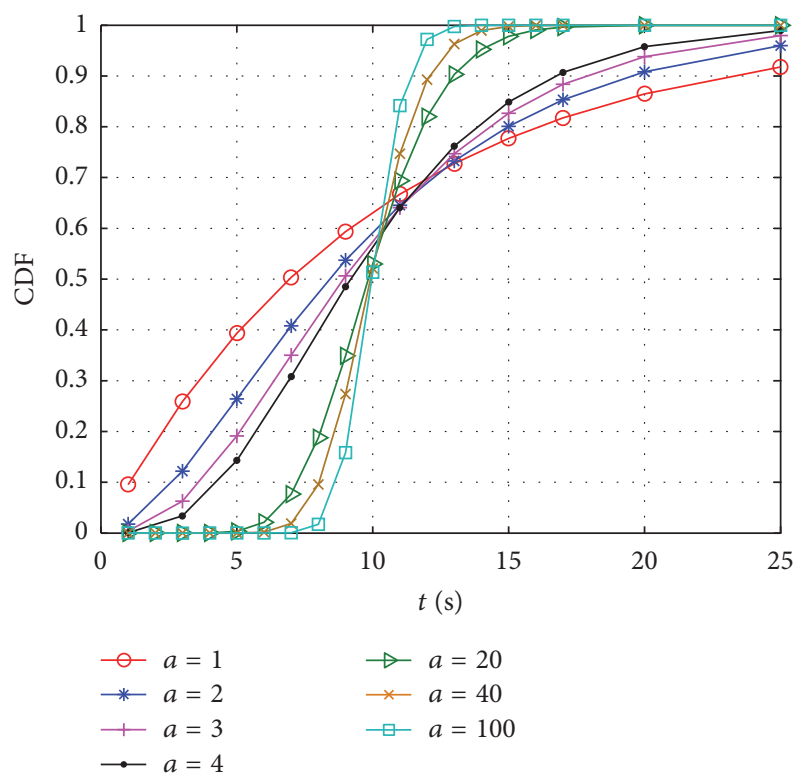

FIgURE 2: The CDFs of mobility model.

distribution function (CDF) can be expressed as

$$
F(t ; q, a)=1-\sum_{k=1}^{a} \frac{(a q t)^{k-1}}{(k-1) !} e^{-a q t} .
$$

As an example, the PDFs with a mean time headway of $\bar{t}=10 \mathrm{~s}$ and $q=0.1$ are shown in Figure 1, where $t$ disperses from $1 \mathrm{~s}$ to $25 \mathrm{~s}$ and $a=1,2,3,4,20,40$ and 100 typically. As $a$ increases, the distribution varies from an exponential distribution to a normal distribution and converges around $10 \mathrm{~s}$, gradually.

Figure 2 shows the corresponding CDFs and the CDFs approach to 1 at $t=25 \mathrm{~s}$ when $a=4$ and $t=13 \mathrm{~s}$ when 


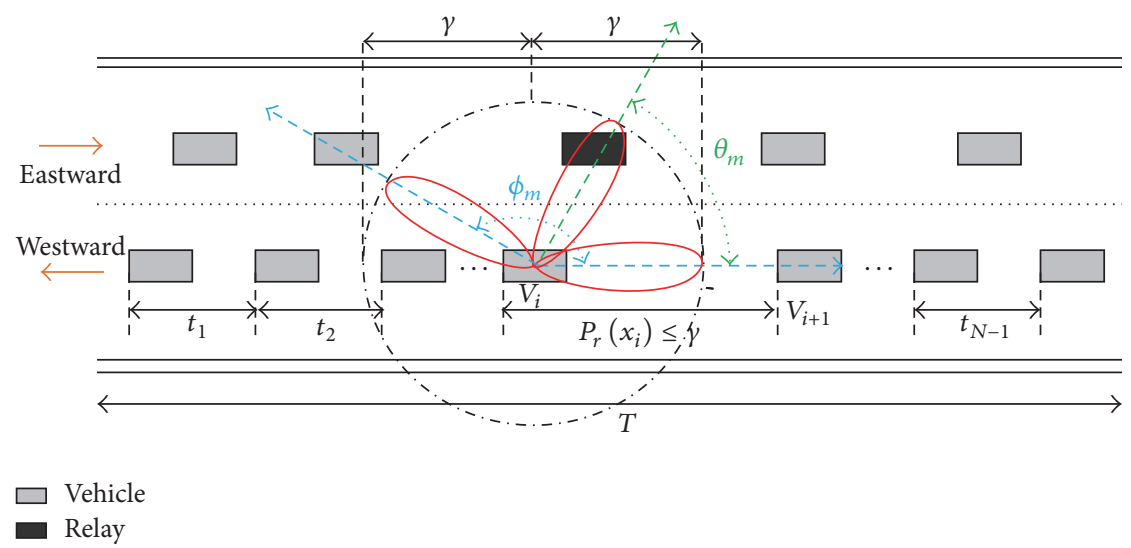

FIGURE 3: The two-way highway model with beamforming technology.

$a=100$. These curves show that the CDFs ascend abruptly at $t=10 \mathrm{~s}$ in the case of a larger value of $a$.

From Figures 1 and 2, we can observe that the mobility model with $a=1$ represents the sparse traffic, and the traffic becomes denser as $a$ increases. The mobility model also represents the traffic density from off-peak hours to rush hours.

3.2. Free-Space Path Loss Model. Free-space path loss model is adopted, which can be calculated as follows:

$$
P_{r}(x)(\mathrm{dBm})=\frac{P_{t}(\mathrm{dBm}) G_{t} G_{r} \lambda^{2}}{(4 \pi)^{2} x^{2}},
$$

where $P_{r}(x)$ is the input of the receiving antenna, $P_{t}$ is the output power to the transmitting antenna, $G_{t}$ and $G_{r}$ are the antenna gains of the transmitting and receiving antennas respectively, and $\lambda$ is the wavelength, and the distance, $x$, between the antennas is calculated by

$$
x=\bar{v} t
$$

where $\bar{v}$ is the mean velocity of vehicles.

For simplicity, we assume that $G_{r}=0 \mathrm{~dB}$ and (3) can be expressed further as

$$
\begin{aligned}
P_{r}(x)(\mathrm{dBm})= & P_{t}(\mathrm{dBm})+G_{t}(\mathrm{~dB}) \\
& +20 \log _{10}\left(\frac{c}{4 \pi x f}\right),
\end{aligned}
$$

where $f$ is the signal frequency and $c$ is the light speed. In general, $G_{t}$ can be expressed as

$$
G_{t}=\eta D(\theta)
$$

where $\eta$ is antenna's efficiency and $D(\theta)$ is directivity. We further assume that $\eta=1$. At transmitter, if isotropic antenna is used, $D=1$ at this moment. When beamforming antenna array is adopted, the gain of an antenna maximizes in its direction, resulting in $G_{t}=D_{\max }$. For example, the maximum value of $D$ is $1.64(2.15 \mathrm{~dB})$ for a half-wave dipole antenna.
The distance, $d_{0}$, is used to as a reference for received power. (5) is thus rewritten as

$$
\begin{aligned}
P_{r}(x)(\mathrm{dBw})= & 10 \log _{10} P_{r}\left(d_{0}\right)+20 \log _{10}\left(\frac{d_{0}}{x}\right) \\
& +G_{t}(\mathrm{~dB}) .
\end{aligned}
$$

The probability of received signal with a power level exceeding a threshold of $\gamma$ is given as

$$
P_{\mathrm{Q}}\left(x_{i}\right)=\operatorname{Pr}\left[P_{r}\left(x_{i}\right)>\gamma\right]=Q\left(\gamma-P_{r}\left(x_{i}\right)\right),
$$

where $\gamma$ can be calculated as

$$
\gamma(\mathrm{dBw})=10 \log _{10} P_{r}\left(d_{0}\right)+20 \log _{10}\left(\frac{d_{0}}{x_{0}}\right),
$$

where $x_{0}=\bar{v} t_{0}$, where $t_{0}$ is time headway threshold, also known as communication range, and $\bar{v}$ is the mean velocity of vehicles. The probability of that $P_{r}\left(x_{i}\right)>\gamma$ is computed from (7)-(9) as

$$
P_{\mathrm{Q}}\left(x_{i}\right)=Q\left(20 \log \frac{x_{i}}{x_{0}}-G_{t}(\mathrm{~dB})\right)
$$

which is the connectivity probability between two vehicles spanned by a distance of $x_{i}$.

\section{Connectivity Analysis}

The connectivity probability is mainly determined by traffic density and intervehicle time headway. Figure 3 demonstrates the mobility model in two-way highway where the total time is $\mathrm{T}$ and the westward highway is divided into $N-1$ time headway numbered as $t_{1}, t_{2}, \ldots, t_{N-1}$, respectively. Each $t_{i}$ is considered as independent of each other and the rate of flow $q=N / T$.

In two-way highway, two vehicles may connect to each other with the help of the vehicle in neighboring lanes, if any, either in the same or opposite direction, which is illustrated in Figure 3 [21]. $V_{i+1}$ cannot connect with $V_{i}$ since $P_{r}\left(x_{i}\right) \leq \gamma$. However, the vehicle in dark gray located within the distance 
of $2 \gamma$ centered at $V_{i}$ in the eastward lane can store and forward the messages to $V_{i+1}$.

Moreover, beamforming antenna array with beam directions is adopted in each vehicle, which are presented to show individual signal paths in above mobility model, where $\theta_{m}$ represents the beam direction and $\phi_{m}$ is the maximum angle of scanning range for $V_{i}$.

The vehicles on the eastward lane following Poisson distribution are assumed, and the probability that there are $n$ relay vehicles within $\left(0,2 t_{0}\right)$ is given by

$$
P_{V}=\operatorname{Pr}\left[n \text { vehicles in }\left(0,2 t_{0}\right)\right]=\frac{\left(2 t_{0} q_{0}\right)^{n}}{n !} e^{-2 t_{0} q_{0}} \text {, }
$$

where $q_{0}$ is the arrival rate on the eastward highway.

The probability of that there are no relay vehicles arriving in the $2 t_{0}$ interval is obtained from (11)

$$
P_{V 0}=P_{V}(n=0)=e^{-2 t_{0} q_{0}} .
$$

By considering path loss, the computation of the connectivity probability becomes complicated as different segments have different probabilities varying with the distance. There are $C_{N-1}^{m}$ combinational cases for $m$ random connected segments in $N-1$ different segments.

Set $\mathbf{A}$ represents all series numbers of the time headway segments and the subset, $\mathbf{B}_{\mathbf{k}}$, represents $m$ random series numbers of $\mathbf{A}$. There are $C_{N-1}^{m}$ combinational cases for $m$ connected elements in $N-1$ different elements. That is, $\mathbf{A}=$ $\{1,2, \ldots, N-1\}$ and $\mathbf{B}_{\mathbf{k}}=\left\{a_{k, 1}, a_{k, 2}, \ldots, a_{k, m}\right\}, \mathbf{B}_{\mathbf{k}} \subseteq \mathbf{A}$, where $m$ is an integer, $0 \leq m \leq N-1$, and $k=1,2, \ldots, C_{N-1}^{m}$. Each $\mathbf{B}_{\mathbf{k}}$ contains $m$ elements, and the number of $\mathbf{B}_{\mathbf{k}}$ is $C_{N-1}^{m}$ in each case. Set $\mathbf{D}_{\mathbf{k}}$ to represent $(N-1-m)$ unconnected segments. It is a complementary set of $\mathbf{B}_{\mathbf{k}}$ in the set $\mathbf{A}$. That is $\mathbf{D}_{\mathbf{k}}=C_{A} \mathbf{B}_{\mathbf{k}}$ or $\mathbf{D}_{\mathbf{k}}=\left\{a_{k, m+1}, a_{k, m+2}, \ldots, a_{k, N-1}\right\}$. The number of $\mathbf{D}_{\mathbf{k}}$ is $C_{N-1}^{m}$ in every $m$, and each $\mathbf{D}_{\mathbf{k}}$ contains $N-1-m$ elements.

The connectivity probability is that there are $m$ segments that are connected and $N-1-m$ segments that are not connected, which can be given as

$$
\begin{aligned}
& P_{M Q}= \operatorname{Pr}[m \mid(N-1) \text { arrivals in }(0, t)] \\
&=\sum_{k=1}^{C_{N-1}^{m}}\left\{\prod_{i=1}^{m} P_{Q}\left(x_{B_{k}(i)}\right) \cdot \prod_{j=1}^{N-1-m} P_{S}\left(x_{D_{k}(j)}\right)\right\}, \\
& \quad m=0,1, \ldots, N-1,
\end{aligned}
$$

where $B_{k}(i)$ and $D_{k}(j)$ are the series numbers of $m$ connected and $N-1-m$ unconnected segments. $x_{B_{k}(i)}$ and $x_{D_{k}(j)}$ are the corresponding distances of segments. $P_{\mathrm{Q}}\left(x_{B_{k}(i)}\right)$ is the probability of each one in $m$ connected segments in (10), and $P_{S}\left(x_{D_{k}(j)}\right)$ which is the probability of each one in $N-1-m$ unconnected segments, can be calculated by

$$
P_{S}\left(x_{D_{k}(j)}\right)=1-P_{Q}\left(x_{B_{k}(i)}\right)
$$

TABLE 1: Parameters used in simulations.

\begin{tabular}{lc}
\hline Parameters & Default value \\
\hline Total time & $T=3600 \mathrm{~s}$ \\
Rate of vehicle flow & $0.05 \leq q \leq 1$ \\
Time headway threshold & $t_{0}=15 \mathrm{~s}$ and $25 \mathrm{~s}$ \\
Rate of relay flow & $q_{0}=0.1$ \\
Mean velocity & $\bar{v}=20 \mathrm{~m} / \mathrm{s}$ \\
Signal frequency & $f=5.9 \mathrm{GHz}$ \\
Transmitted power & $P_{t}=27 \mathrm{dBm}$ \\
Gain of transmitting antenna & $G_{t}=2.15 \mathrm{~dB}$ \\
Reference distance & $d_{0}=10 \mathrm{~m}$ \\
Reference received power & $P_{r}\left(d_{0}\right)=-40.86 \mathrm{dBm}$ \\
Received power threshold & $P_{r}(300)=-70.40 \mathrm{dBm}$ \\
Received power threshold & $P_{r}(500)=-74.84 \mathrm{dBm}$ \\
\hline
\end{tabular}

According to Bernoulli trials, the connectivity probability in two-way highway can be derived as

$$
\begin{aligned}
P_{c} & =\sum_{m=0}^{N-1}\left[\left(1-P_{V 0}\right)^{N-1-m} P_{M Q}\right] \\
& =\sum_{m=0}^{N-1}\left[\left(1-e^{-2 t_{0} q_{0}}\right)^{N-1-m}\right. \\
& \left.\cdot \sum_{k=1}^{C_{N-1}^{m}}\left\{\prod_{i=1}^{m} P_{\mathrm{Q}}\left(x_{B_{k}(i)}\right) \cdot \prod_{j=1}^{N-1-m} P_{S}\left(x_{D_{k}(j)}\right)\right\}\right] .
\end{aligned}
$$

\section{Simulations and Analysis}

Simulations are conducted to validate the above theoretical study, and the related parameters are listed in Table 1.

We assume that $N$ vehicles are randomly placed on a lane according to the Erlang distribution with $a=1,2,3,4,20,40$ and 100 typically, and the half-wave dipole antennas are chosen with the maximum gain of $2.15 \mathrm{~dB}$.

Figures 4-7 illustrate the connectivity probability based on the above proposed models. The approximate connectivity probability calculated from (15) is expressed with solid and dashed lines, while simulation ones are expressed in discrete symbol. The simulation and analysis results of the connectivity probability are presented, which fit well where the values of shape parameter $(a=1,2,3,4,20,40$ and 100) in Erlang mobility model and two values of the time headway $\left(t_{0}=15 \mathrm{~s}\right.$ and $t_{0}=25 \mathrm{~s}$ ) are considered. The connectivity probability sharply grows with $q$, and a moderate $q$ is sufficient to attain reasonable performance.

In Figure 4, the value of a represents diverse traffic density. The connectivity probability achieves its lowest value in the case of $a=1$ which represents the sparse traffic. As $a$ increases, the traffic becomes denser gradually, and the connectivity probability grows.

In Figure 5, it is observed that the connectivity probability is all improved when adopting beamforming technology 


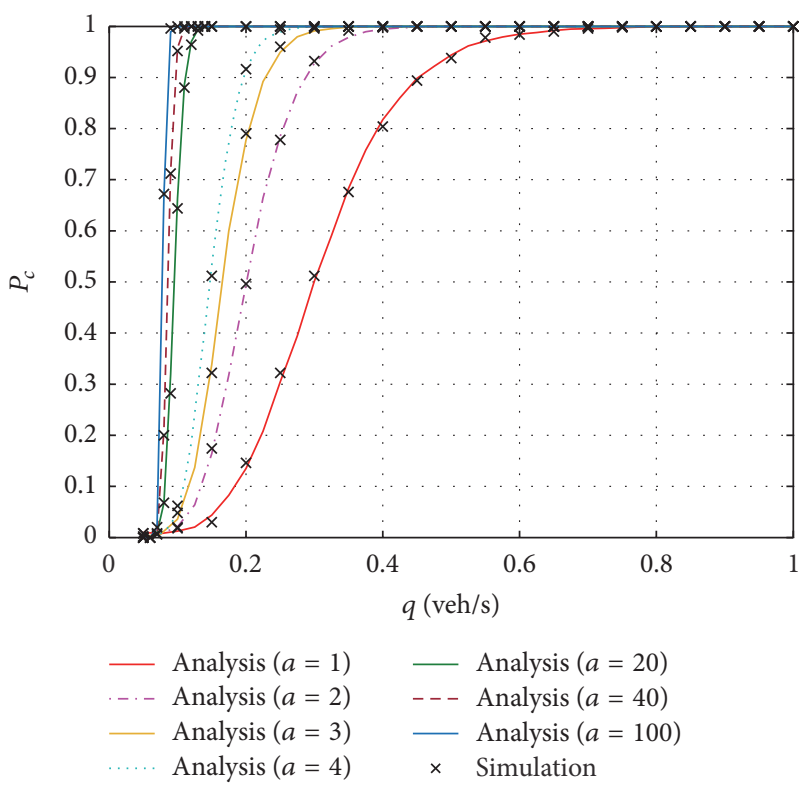

FIGURE 4: Connectivity probability varies with shape parameter $(a=$ $1,2,3,4,20,40$ and 100) in Erlang mobility model where $t_{0}=15 \mathrm{~s}$.

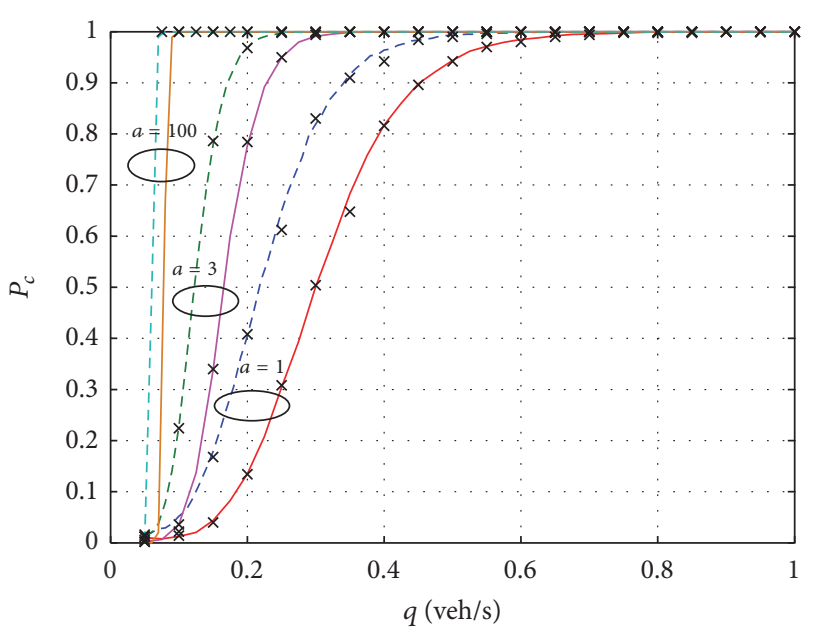

FIGURE 5: Connectivity probability with or without beamforming where $t_{0}=15 \mathrm{~s}$ and $a=1,3$ and 100 .

under different $a$. Beamforming antenna array is proven to be able to enhance connectivity and prolong connecting distance between adjacent vehicles.

In Figures 6 and 7, the connectivity in the case of $t_{0}=$ $25 \mathrm{~s}$ outperforms that in the case of $t_{0}=15 \mathrm{~s}$ with or without beamforming. A large value of $t_{0}$ guarantees a well-connected network for various traffic densities than a small value of $t_{0}$.

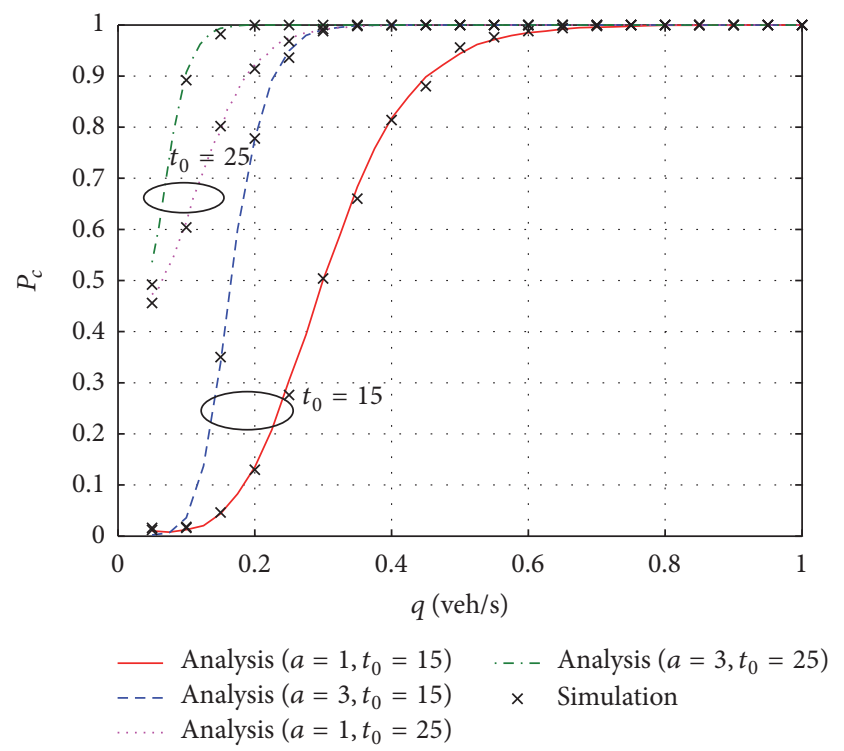

FIGURE 6: Connectivity probability varies with time headway of $t_{0}=$ $15 \mathrm{~s}$ and $25 \mathrm{~s}$ without beamforming.

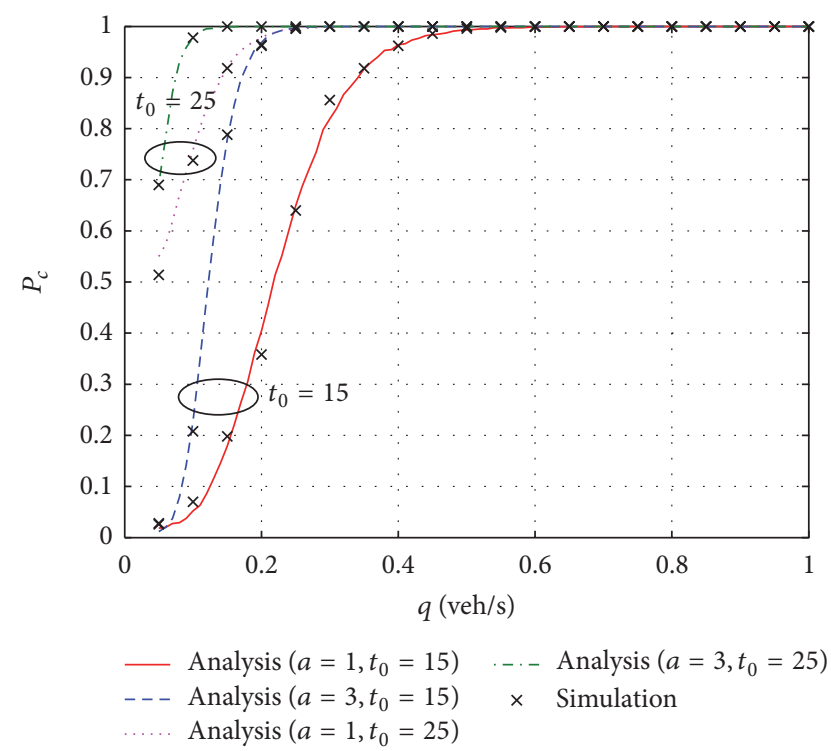

FIgURE 7: Connectivity probability varies with time headway of $t_{0}=$ $15 \mathrm{~s}$ and $25 \mathrm{~s}$ with beamforming.

\section{Conclusion}

In this paper, the connectivity probability is researched in two-way highway when adopting beamforming antenna array. The Erlang mobility model can depict the behavior of vehicles in sparse and dense scenarios. The effort demonstrates that the rate of traffic flow as well as the time headway heavily impacts connectivity. On the other hand, the path loss among vehicle communication can be compensated by using beamforming antenna array, resulting in prolonged connection distance. The connectivity probability can be used as metric for relevant physical and network layer study in 
broad range of topics ranging from latency estimate to realtime routing.

\section{Conflicts of Interest}

The authors declare that they have no conflicts of interest.

\section{Acknowledgments}

This work is supported by NSFC 61571282 and State Key Laboratory of Rail Traffic Control and Safety RCS2017K012.

\section{References}

[1] T. Willke, P. Tientrakool, and N. Maxemchuk, "A survey of intervehicle communication protocols and their applications," IEEE Communications Surveys and Tutorials, vol. 11, no. 2, pp. 3-20, 2009.

[2] D. B. Rawat, B. B. Bista, G. Yan, and S. Olariu, "Vehicle-tovehicle connectivity and communication framework for vehicular ad-hoc networks," in Proceedings of the 8th International Conference on Complex, Intelligent and Software Intensive Systems (CISIS '14), pp. 44-49, July 2014.

[3] S. Kwon, Y. Kim, and N. B. Shroff, "Analysis of connectivity and capacity in 1-D vehicle-to-vehicle networks," IEEE Transactions on Wireless Communications, vol. 15, no. 12, pp. 8182-8194, 2016.

[4] X. Hou, Y. Li, D. Jin, D. O. Wu, and S. Chen, "Modeling the impact of mobility on the connectivity of vehicular networks in large-scale urban environments," IEEE Transactions on Vehicular Technology, vol. 65, no. 4, pp. 2753-2758, 2016.

[5] J. Harri, F. Filali, and C. Bonnet, "Mobility models for vehicular ad hoc networks: a survey and taxonomy," IEEE Communications Surveys and Tutorials, vol. 11, no. 4, pp. 19-41, 2009.

[6] A. F. Molisch, F. Tufvesson, J. Karedal, and C. F. Mecklenbräuker, "A survey on vehicle-to-vehicle propagation channels," IEEE Wireless Communications, vol. 16, no. 6, pp. 12-22, 2009.

[7] M. Khabazian and M. K. M. Ali, "A performance modeling of connectivity in vehicular Ad Hoc networks," IEEE Transactions on Vehicular Technology, vol. 57, no. 4, pp. 2440-2450, 2008.

[8] S. Panichpapiboon and W. Pattara-Atikom, "Connectivity requirements for self-organizing traffic information systems," IEEE Transactions on Vehicular Technology, vol. 57, no. 6, pp. 3333-3340, 2008.

[9] P. Golmohammadi, P. Mokhtarian, F. Safaei, and R. Raad, "An analytical model of network connectivity in vehicular ad hoc networks using spatial point processes," in Proceedings of the 15th IEEE International Symposium on a World of Wireless, Mobile and Multimedia Networks (WoWMoM '14), pp. 1-6, IEEE, Sydney, Australia, June 2014.

[10] N. Wisitpongphan, F. Bai, P. Mudalige, V. Sadekar, and O. Tonguz, "Routing in sparse vehicular ad hoc wireless networks," IEEE Journal on Selected Areas in Communications, vol. 25, no. 8, pp. 1538-1556, 2007.

[11] M. Gramaglia, P. Serrano, J. A. Hernández, M. Calderon, and C. J. Bernardos, "New insights from the analysis of free flow vehicular traffic in highways," in Proceedings of the IEEE International Symposium on a World of Wireless, Mobile and Multimedia Networks (WoWMoM '11), pp. 1-9, Lucca, Italy, June 2011.
[12] M. Gramaglia, O. Trullols-Cruces, D. Naboulsi, M. Fiore, and M. Calderon, "Vehicular networks on two Madrid highways," in Proceedings of the 11th Annual IEEE International Conference on Sensing, Communication, and Networking (SECON '14), pp. 423-431, Singapore, July 2014.

[13] D. R. Cox and P. A. Lewis, The Statistical Analysis of Series of Events, John Wiley \& Sons, Hoboken, NJ, USA, 1966.

[14] D. Gross and C. M. Harris, Fundamentals of Queueing Theory, John Wiley \& Sons, Hoboken, NJ, USA, 1974.

[15] Y. Fang, I. Chlamtac, and Y.-B. Lin, "Channel occupancy times and handoff rate for mobile computing and PCS networks," IEEE Transactions on Computers, vol. 47, no. 6, pp. 679-692, 1998.

[16] Y.-B. Lin and I. Chlamtac, "Effects of Erlang call holding times on PCS call completion," IEEE Transactions on Vehicular Technology, vol. 48, no. 3, pp. 815-823, 1999.

[17] H. Mott, Remote Sensing with Polarimetric Radar, Wiley-IEEE Press, Hoboken, NJ, USA, 2007.

[18] J. Zhao, Y. Chen, and Y. Gong, "Study of connectivity probability of vehicle-to-vehicle and vehicle-to-infrastructure communication systems," in Proceedings of the 83rd IEEE Vehicular Technology Conference (VTC Spring '16), pp. 1-4, IEEE, Nanjing, China, May 2016.

[19] C. Shao, S. Leng, Y. Zhang, A. Vinel, and M. Jonsson, "Performance analysis of connectivity probability and connectivityaware MAC protocol design for platoon-based VANETs," IEEE Transactions on Vehicular Technology, vol. 64, no. 12, pp. 55965609, 2015.

[20] D. Eckhoff, A. Brummer, and C. Sommer, "On the impact of antenna patterns on VANET simulation," in 2016 IEEE Vehicular Networking Conference (VNC), pp. 1-4, Columbus, OH, USA, December 2016.

[21] A. Kesting, M. Treiber, and D. Helbing, "Connectivity statistics of store-and-forward intervehicle communication," IEEE Transactions on Intelligent Transportation Systems, vol. 11, no. 1, pp. 172-181, 2010. 


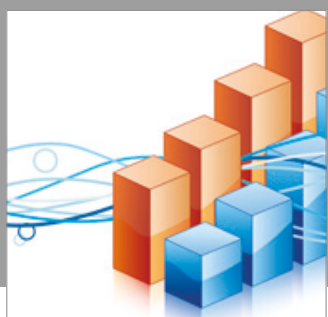

Advances in

Operations Research

vatersals

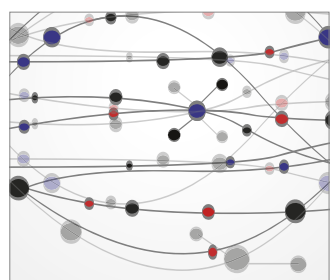

\section{The Scientific} World Journal
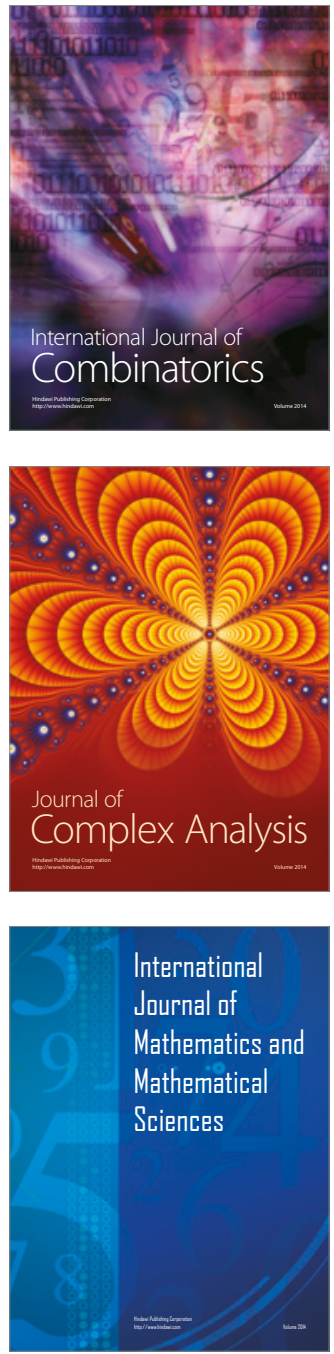
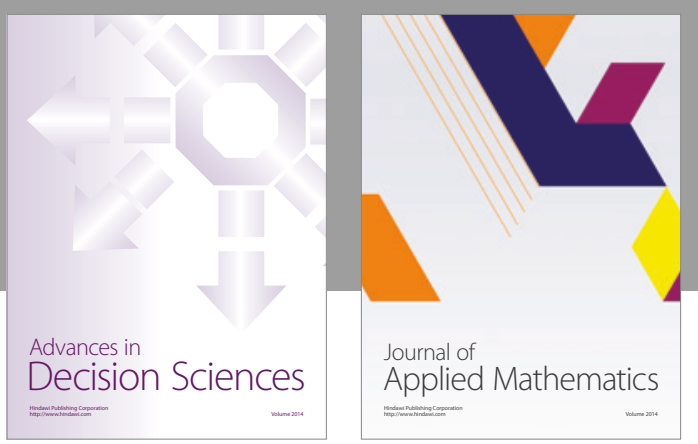

Algebra

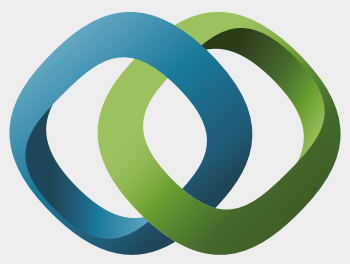

\section{Hindawi}

Submit your manuscripts at

https://www.hindawi.com
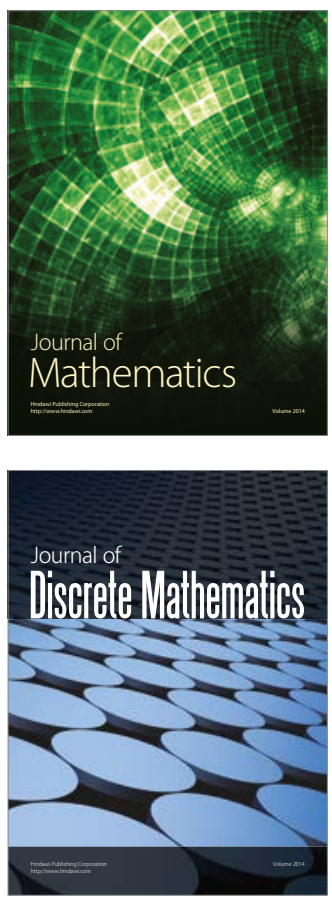

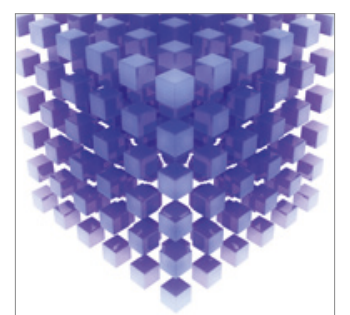

Mathematical Problems in Engineering
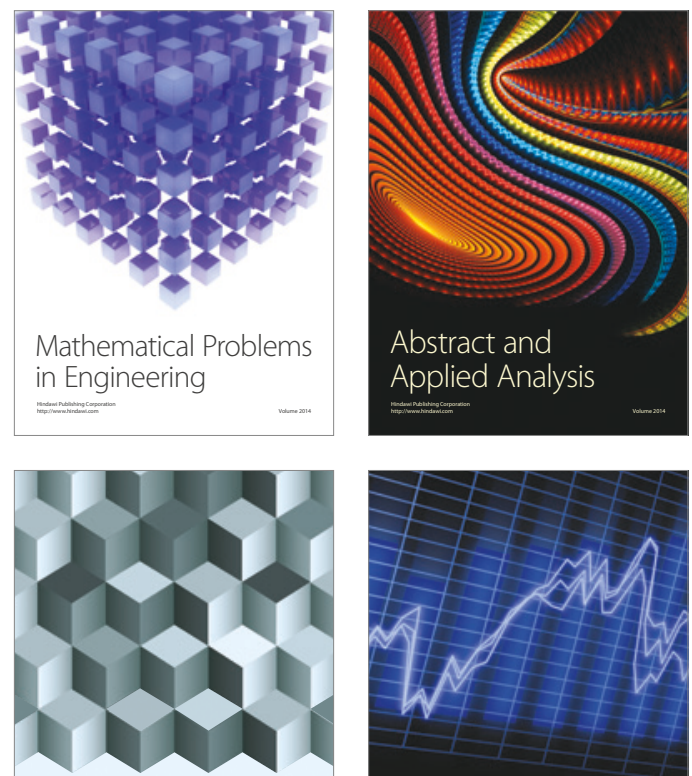

Journal of

Function Spaces

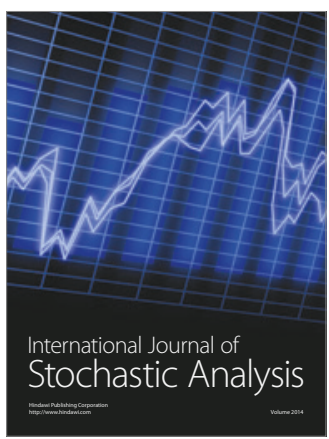

Probability and Statistics
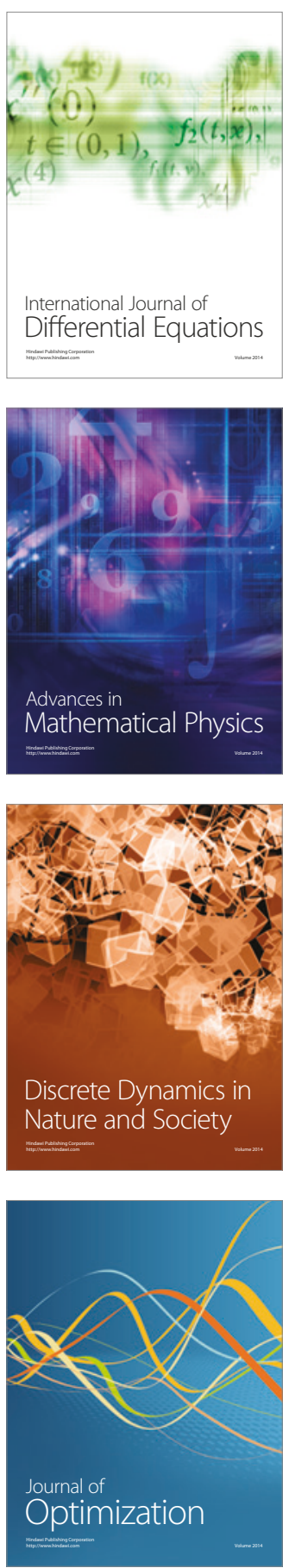